\title{
Review of the NAEPP 2007 Expert Panel Report (EPR-3) on Asthma Diagnosis and Treatment Guidelines
}

\author{
Frank L. Urbano, MD, FACP, for a panel of 9 asthma disease specialists
}

\begin{abstract}
BACKGROUND: In 1989, the National Asthma Education and Prevention Program (NAEPP) convened an expert panel to develop a report that would provide a general approach to the treatment of asthma. Expert Panel Report: Guidelines for the Diagnosis and Management of Asthma, or EPR-1, was published in 1991 and was subsequently updated with 2 other reports, EPR-2 in 1997 and the EPR update in 2002. Advances in science and a greater understanding of the pathophysiology of asthma prompted the NAEPP to convene a 3rd expert panel in 2004. After nearly 3 years of work, Expert Panel Report 3: Guidelines for the Diagnosis and Management of Asthma-Full Report 2007, or EPR-3, was released on August 29, 2007. EPR-3 update from the NAEPP provides health care professionals with new information to improve the care of patients with asthma, including (1) more comprehensive discussion of asthma severity with expanded descriptions of impairment and risk, (2) increased focus on asthma control as a goal of therapy, and (3) expanded discussion of pharmacologic therapy for asthma with updated treatment algorithms.
\end{abstract}

OBJECTIVES: To (1) extract key educational messages from the EPR-3 update that effectively summarize the appropriate management of the patient with asthma and (2) provide supporting literature to substantiate the development of these educational messages.

METHODS: A consensus meeting of 9 asthma experts (4 pharmacists and 5 physicians) was held to discuss the EPR- 3 update and condense its content into a usable format for the health care professional. Experts were selected on the basis of several criteria, including (1) affiliation with the NAEPP, (2) expertise in asthma management, and (3) familiarity with managed care processes. The author served as the 10th member and moderator of the meeting.

RESULTS: Thorough review of the EPR-3 update resulted in the development of 7 key educational messages that can assist the health care professional in improving the management of the patient with asthma. Each educational message is presented with supporting literature to substantiate its distinction as a key point to be referenced when developing protocols for asthma management within managed care organizations.

CONCLUSION: The complexity of asthma and its treatment has necessitated the development of several guidelines from the NAEPP, with the most recent EPR-3 update being released in late August 2007. One expert consensus has distilled the EPR-3 document into 7 key educational messages that can assist the health care professional in improving the care of the patient with asthma.

J Manag Care Pharm. 2008;14(1):41-49

Copyright $\odot$ 2008, Academy of Managed Care Pharmacy. All rights reserved.

\section{What is already known about this subject}

- The NAEPP has produced 2 prior expert reports and 1 update report that have addressed the diagnosis and management of patients with asthma.

- Greater knowledge of the pathophysiology of asthma has necessitated the development of another guideline update, EPR-3.

\section{What this study adds}

- EPR-3 differs from the previous asthma diagnosis and management guidelines in

- providing an expanded discussion on the use of spirometry and the concept of airflow reversibility;

- placing a stronger emphasis on the use of the written asthma action plan;

- adding immunomodulatory therapy (i.e., omalizumab) as an option for certain patients with allergies and severe persistent asthma that is inadequately controlled with the combination of high-dose inhaled corticosteroids (ICSs) and long-acting beta-2 agonists (LABAs);

- providing equal weight to increasing the dose of an ICS or adding a LABA in patients with moderate persistent asthma or asthma that is not controlled on a low-dose ICS;

- expanding the discussion of asthma severity to include the domains of current impairment and future risk;

- greatly expanding the discussion of asthma control as a target of asthma therapy; and

- making several changes to the stepwise approach to managing asthma and to managing asthma exacerbations.
A sthma is a chronic inflammatory disease of the airways that causes a high burden on the global health care system. In the United States alone, approximately 15.7 million adults and 6.7 million children have asthma, ${ }^{1}$ and in 2004, approximately 3,780 patients died from asthma and its complications. ${ }^{2}$ Direct costs of asthma were estimated to be $\$ 11.5$ billion in 2004, with the largest components of cost being prescription drugs and hospital care. ${ }^{3}$

Despite advances in therapy, asthma remains a disease that, in many patients, is not optimally controlled. Patient surveys show that approximately $60 \%$ of people with moderate persistent 
TABLE 7 Key Points Identified by 9 Asthma Experts in Review of the NAEPP EPR-3 (2007) Report on Asthma Diagnosis and Management ${ }^{\mathrm{a}}$

\begin{tabular}{|c|c|}
\hline 1. & Establishing an accurate diagnosis is essential. \\
\hline 2. & Successful management depends on a comprehensive approach. \\
\hline 3. & Assessment of severity determines initial therapy. \\
\hline 4. & Monitoring control determines ongoing therapy. \\
\hline 5. & A stepwise approach should be used for initial and ongoing therapy. \\
\hline 6. & Effective control includes managing special situations. \\
\hline 7. & Managing exacerbations is an important part of asthma care. \\
\hline \multicolumn{2}{|r|}{$\begin{array}{l}\text { a Nine asthma disease experts convened June 7-8, 2007, to review Expert Panel } \\
\text { Report 3: Guidelines for the Diagnosis and Management of Asthma (2007) from } \\
\text { the NAEPP. Available at: www.nhlbi.nih.gov/guidelines/asthma/asthgdln.pdf. } \\
\text { EPR-3 = Expert Panel Report 3; NAEPP = National Asthma Education and } \\
\text { Prevention Program. }\end{array}$} \\
\hline
\end{tabular}

asthma and 33\% of people with severe persistent asthma consider their asthma to be well controlled or completely controlled, which indicates that many patients overestimate their personal level of asthma control. ${ }^{4}$ In addition, studies have shown that providers also tend to overestimate a patient's level of asthma control, which suggests a need for further education on asthma management. ${ }^{5}$

In 1989, the National Asthma Education and Prevention Program (NAEPP) convened an expert panel to develop a report that would provide a general approach to the treatment of asthma. This report, Expert Panel Report: Guidelines for the Diagnosis and Management of Asthma, or EPR-1, was published in 1991 and was subsequently updated with 2 other reports, EPR-2 in $1997^{6}$ and EPR update in 2002. ${ }^{7}$

Because of advances in science and an increasing understanding of the pathophysiology of asthma, the NAEPP convened a Third Expert Panel to discuss updating the existing asthma guidelines in 2004. After nearly 3 years of work, Expert Panel Report 3: Guidelines for the Diagnosis and Management of AsthmaFull Report 2007, or EPR-3, was officially released on August 29, 2007. ${ }^{8}$

EPR-3 is a comprehensive document that discusses the definition, pathophysiology, and pathogenesis of asthma; the long-term management of asthma; the management of asthma in special populations; and the management of asthma exacerbations. In addition, the 4 components of asthma management, which have been stressed in all versions of the guidelines, are discussed and include measures of asthma assessment and monitoring; education for a partnership in asthma care; control of environmental factors and comorbid conditions that affect asthma; and pharmacologic therapy for asthma. The final document, including table of contents, methodology, and references, is 487 pages long.
While reviewing the entire document is certainly possible and is obviously desirable, it is probably impractical for the average health care professional. The imposing size of the EPR-3 document precipitated the conveying of a meeting of 9 asthma experts (including 1 member of the NAEPP Coordinating Committee, 1 member of the Third Expert Panel, and 1 consultant reviewer for EPR-3) on June 7-8, 2007, to discuss the forthcoming guidelines and to extract from them the key points judged to be the most important and clinically relevant. The major differences between EPR-3 and the previous versions of the asthma guidelines were discussed. The result of that discussion in this group of 9 asthma disease experts was the creation of 7 key points that summarize the content of the guidelines (Table). These 7 key points and their associated scientific rationale are discussed below.

\section{Establishing an Accurate Diagnosis Is Essential}

Clinicians should consider the diagnosis of asthma when patients present with episodic symptoms of airflow obstruction that is at least partially reversible, and when alternative diagnoses have been excluded. ${ }^{8}$ Indicators for a diagnosis of asthma include wheezing, cough, chest tightness, dyspnea, worsening of symptoms in the presence of environmental stimuli, and worsening of symptoms at night. Diagnosis of asthma is established through the use of medical history, physical examination, and spirometry.

All versions of the asthma guidelines have used the aforementioned approach in the diagnosis of asthma. EPR-3 places a strong focus on the use of spirometry, which is recommended both before and after the inhalation of a short-acting bronchodilator in all patients suspected of having asthma. Studies have shown that while history and physical examination can provide clues to the diagnosis of asthma, objective measures of lung function, such as spirometry, are necessary for the accurate diagnosis of asthma. ${ }^{9}$ EPR-3 also discusses the concept of reversibility in further detail, indicating that some patients who have signs and symptoms of asthma may not initially demonstrate reversibility on spirometry. In these patients a short course of oral corticosteroid therapy may be required to improve their asthma control in order to demonstrate reversibility.

Many other clinical disorders may mimic asthma, and therefore other diagnostic possibilities should be considered in the patient presenting with signs and symptoms suggestive of asthma. Conditions to be considered include allergic rhinitis and sinusitis; congestive heart failure; pulmonary embolism; chronic obstructive pulmonary disease; drug-related cough; and other pulmonary conditions. EPR-3 specifically adds a discussion on cough-variant asthma and vocal cord dysfunction as potential disorders that may present similarly to classical asthma. A careful diagnostic workup for asthma should always include consideration of the diagnostic entities mentioned above. 


\section{Successful Management Depends on a Comprehensive Approach}

Management of the patient with asthma requires an approach that considers many factors. In previous versions of the guidelines, as well as in EPR-3, a comprehensive approach has been stressed, including education, control of environmental factors, and use of appropriate pharmacologic therapies. Education should begin early and involve all members of the health care team delivering the same key message to the patient. Patients should be taught what asthma is and what defines well-controlled asthma; the roles of the different medications used to treat asthma; the proper use of an inhaler; how to recognize worsening asthma; when and where to seek additional care when necessary; and methods to control environmental exposures and triggers. EPR-3 places a stronger emphasis on the written asthma action plan, which should include providing instructions for daily management and recognizing and handling worsening asthma, including adjusting the dose of medications. The evidence supporting the use of such written plans is inconclusive, but they are generally believed to be beneficial in preventing or managing asthma exacerbations. ${ }^{8}$ Education of providers who treat patients with asthma is also stressed, although studies are once again inconclusive. In general, EPR-3 recommends that provider education be multifaceted and involve interactive learning strategies, on the basis of studies that show significant long-term benefits of such education on the quality of asthma care. ${ }^{10}$

Controlling environmental factors improves long-term management of asthma. Methods that can be used to achieve control of environmental factors include reducing or eliminating exposure to allergens (e.g., animal dander, cockroaches) and indoor/outdoor pollutants (e.g., perfumes, volatile organic compounds), as well as stopping smoking, including by others who live in the home. As with education, this should involve a multifaceted approach, since programs that focus on educating patients and providing tools for reducing environmental exposures have demonstrated success in reducing asthma morbidity. ${ }^{8}$

Appropriate pharmacologic therapy for asthma is the cornerstone of its management. All versions of the guidelines have acknowledged the key distinction between long-term controller medications and short-term quick-relief medications. Inhaled corticosteroids (ICSs) are still considered the most potent and consistently effective long-term control medications for asthma. They are more effective than any other class of controller medications, and they are safe and well tolerated. Cromolyn sodium, nedocromil, inhaled long-acting beta-2 agonists (LABAs), leukotriene modifiers, theophylline, and omalizumab are all considered possible adjunctive therapies to ICS therapy. The 2 major changes in EPR-3 with regard to pharmacologic therapy include (1) the addition of immunomodulators, specifically anti-IgE (omalizumab) therapy, for patients with severe persistent asthma and allergies, and (2) equal weight given to increase the ICS dose or the option of adding a LABA in patients with moderate persistent asthma or asthma inadequately controlled on a low-dose ICS. ${ }^{8}$

The immunomodulatory agent omalizumab is a humanized monoclonal antibody to the Fc portion of the IgE antibody, which prevents IgE from binding to its receptor on mast cells and basophils and consequently inhibits the release of allergic mediators. Since asthma and atopy have been linked, ${ }^{11-14}$ an agent such as omalizumab would be expected to have a beneficial effect on asthma control. Studies have shown that use of omalizumab is associated with reductions in asthma exacerbations, ${ }^{15}$ reductions in the dose of ICS needed for control of symptoms, ${ }^{16-17}$ and improvements in quality of life. ${ }^{18}$ EPR-3 recommends that its use be limited to those patients with allergies and severe persistent asthma that are inadequately controlled with the combination of high-dose ICS and LABA, since omalizumab has not yet been compared with other adjunctive therapies in moderate asthma. Anaphylactic reactions have been reported with omalizumab, ${ }^{19}$ and "clinicians who administer omalizumab should be prepared and equipped to identify and treat anaphylaxis that may occur."

LABAs, including salmeterol and formoterol, are effective because of their ability to cause bronchodilation up to 12 hours after administration. EPR-3 recommends that LABAs be used as an adjunct to ICS therapy for providing long-term control of symptoms, and that they are the preferred adjunctive therapy to combine with ICSs in youths $\geq 12$ years of age and adults. ${ }^{8}$ The major change in EPR-3 is that in patients who have asthma not sufficiently controlled with ICS alone, acceptable therapeutic options of equal weight include either (1) increasing the dose of the ICS, or (2) adding a LABA to the ICS. This recommendation is based on a thorough review of the evidence comparing LABA add-on therapy with increasing ICS dose. ${ }^{20}$ EPR-3 also recommends that for patients who have more severe persistent asthma, the combination of LABAs and ICSs should be used as the most effective therapy. Finally, EPR-3 also notes that daily use of LABAs should generally not exceed $100 \mathrm{mcg}$ of salmeterol or $24 \mathrm{mcg}$ of formoterol.

The safety of LABAs was also considered by EPR-3 due to initial postmarketing surveillance that suggested an increase in asthma deaths in patients treated with LABAs. ${ }^{21}$ Subsequent studies provided conflicting results, but 1 large placebo-controlled postmarketing trial of salmeterol added to usual therapy in 2006 found an increased risk of asthma-related deaths and combined asthma-related death or life-threatening experiences in the population treated with LABAs. ${ }^{22}$ For this reason, the U.S. Food and Drug Administration issued a public health advisory regarding the potential risk associated with LABAs in 2006,23 and all products containing a LABA now have a black box warning.

\section{Assessment of Severity Determines Initial Therapy}

Once a diagnosis of asthma has been established, it is important to characterize the severity of the patient's asthma in order to guide the initial therapeutic choice. Severity is defined by EPR-3 as 


\begin{tabular}{|l|l|}
\hline FIGURE 1 & $\begin{array}{l}\text { Asthma Management-Measures of } \\
\text { Severity and Control Include Domains } \\
\text { of Current Impairment and Future Risk }\end{array}$ \\
\hline Components of Control & \\
\hline Impairment & $\begin{array}{l}\text { The frequency and intensity of symptoms } \\
\text { and functional limitations the patient is } \\
\text { experiencing or has recently experienced. }\end{array}$ \\
\hline Risk & $\begin{array}{l}\text { The likelihood of asthma exacerbations, } \\
\text { progressive decline in lung function } \\
\text { (or, for children, reduced lung growth), } \\
\text { or risk of adverse effects from medication. }\end{array}$ \\
\hline
\end{tabular}

Derived from Expert Panel Report 3; $36^{8}$

the intrinsic intensity of the disease process, as measured by the degree of current impairment and the assessment of future risk or by defining the least amount of medication needed to achieve control of symptoms. ${ }^{8}$ While the concept of asthma severity is not a new one and was present in earlier versions of the asthma guideline, it has been refined and expanded in EPR-3 to include the additional concepts of current impairment and future risk.

The term "impairment" refers to the degree to which asthma interferes with the normal functioning of the patient. Domains included in impairment are nighttime awakenings; need for quick-relief medications; work or school days missed; ability to engage in normal activities; quality of life; and lung function as measured by spirometry. Studies have confirmed that these domains are important predictors of general health status, symptoms, limitations in normal daily activities, resource utilization (such as emergency department [ED] visits and hospitalizations), and costs. ${ }^{24-25}$ The term "future risk" refers to the individual risk of asthma exacerbations and death, adverse effects from medications, and progressive loss of lung function (Figure 1). An increased risk for exacerbations or death may be predicted by several factors, including more severe airflow obstruction, ${ }^{26}$ more frequent ED visits or need for intensive care unit care, ${ }^{27}$ depression, ${ }^{28}$ and poorer attitudes about use of asthma medications. ${ }^{29}$

EPR-3 contains 3 tables that can be used to assess asthma severity in children aged 0-4 years, 5-11 years, and $\geq 12$ years. $^{8}$ In this version of the guidelines, the term "mild intermittent" is replaced with the term "intermittent" to emphasize that patients who have intermittent asthma may also have severe exacerbations. ${ }^{8}$ The 3 severity tables in EPR-3 contain the domains of impairment and risk identified previously. On the topic of asthma severity, an important emphasis in EPR-3 is the fact that FEV1/ FVC may be a more sensitive indicator of asthma severity than the other components of the impairment domain in children. ${ }^{30}$ Conversely, FEV1 is suggested as a useful measure of the risk of exacerbations in this age group. ${ }^{31}$

\section{Monitoring Control Determines Ongoing Therapy}

After therapy for asthma has been initiated, it is important to periodically assess and monitor the individual patient's progress to ascertain whether the therapy is effective and the goals of therapy are being met. In previous versions of the guidelines, asthma severity was emphasized more than ongoing monitoring and assessment of asthma control. EPR-3 greatly expands the concept of asthma control as a measure used to determine the effectiveness of asthma therapy. According to EPR-3, asthma control is achieved by considering the same domains that one considers when classifying severity of impairment and risk. Reducing impairment includes preventing chronic and troublesome symptoms, reducing the need for short-acting bronchodilators, maintaining normal or near-normal lung function, maintaining normal or near-normal activity levels, and meeting patient and family expectations of therapy. ${ }^{8}$

Asthma control has been added as a target of guideline-based management of asthma because of observations regarding the effects of asthma control on clinical and other parameters. Studies have shown that patients with well-controlled asthma can have improved quality of life $^{32}$ and decreased health care resource utilization. ${ }^{33}$ The Gaining Optimal Asthma Control (GOAL) study was a randomized, double-blind study of 3,421 patients with uncontrolled asthma. It compared fluticasone propionate and salmeterol/fluticasone in achieving 2 rigorous, composite, guideline-based measures of control: totally and well-controlled asthma. ${ }^{34}$ In the GOAL study, well-controlled asthma was achieved in 33\% to $71 \%$ of patients, while totally controlled asthma was achieved in $8 \%$ to $42 \%$ of patients. Those patients who achieved either well-controlled or totally controlled asthma had a significantly lower rate of exacerbations and significantly higher quality of life scores. These data served to reinforce the importance of achieving asthma control, and EPR-3 refers to the results of this trial when discussing its expanded focus on asthma control.

EPR-3 contains 3 tables that can be used to monitor asthma control in children aged 0-4 years, 5-11 years, and $\geq 12$ years. ${ }^{8}$ These tables contain the previously mentioned domains of asthma control, impairment, and risk, and classify asthma control into 3 categories-well controlled, not well controlled, and very poorly controlled. Individual components that should be considered when classifying the level of asthma control are indicated in Figure 2. When using these tables, clinicians should base the level of control on the most severe impairment or risk category. Ultimately, the level of asthma control should be used to determine if changes to therapy are necessary to improve the patient's control.

\section{A Stepwise Approach Should Be Used} for Initial and Ongoing Therapy

In previous and current versions of the guidelines, a stepwise approach to therapy has been recommended. Using such a 
FIGURE 2 Components of Asthma Control

\begin{tabular}{|c|c|}
\hline Components of Control & Symptoms \\
\hline Impairment & $\begin{array}{l}\text { - Nighttime awakenings } \\
\text { - Interference with normal activity } \\
\text { - Short-acting beta-2 agonist use } \\
\text { for symptom control } \\
\text { - Lung function }{ }^{\mathrm{a}} \\
\text { - Validated questionnaires }\end{array}$ \\
\hline Risk & $\begin{array}{l}\text { - Exacerbations requiring oral systemic } \\
\text { corticosteroids } \\
\text { - Progressive loss of lung function } \\
\text { - Treatment-related adverse effects }\end{array}$ \\
\hline
\end{tabular}

a Not a component of control in children aged 0-4 years.

b Only in patients 12 years old.

"Referred to as "reduction in lung growth" in children aged 5-11 years.

Derived from Figures 3-5a, 3-5b, 3-5c, Expert Panel Report 3; 75-77.

scheme, therapy is initiated after initial severity is determined. Those patients classified with intermittent asthma should be treated with short-acting bronchodilators on an as-needed basis, while those classified with persistent asthma should be treated by initiating the lowest step therapy that will control their symptoms. EPR-3 states that the goal of asthma therapy should be to maintain long-term control of asthma with the least amount of medication, thereby exposing the patient to the least risk for adverse effects from pharmacologic therapy. Accordingly, once therapy is initiated and the level of asthma control is assessed, changes can be made to therapy according to this stepwise approach. This includes step-down therapy as well. ${ }^{8}$

EPR-3 contains 3 tables that may be used to guide the stepwise approach to managing asthma. Unlike previous versions, children have now been divided into 2 age groups, 0-4 years and 5-11 years, while youths and adults $\geq 12$ years remain a separate group. ${ }^{8}$ In addition, EPR-3 now recognizes 6 steps in the stepwise approach rather than 4 in order to simplify the actions in each step.

According to EPR-3, there have been several notable changes to the stepwise approach in comparison with previous guidelines. In the 0-4-year age group, for patients not well controlled on low-dose ICS, increasing the dose of ICS to medium dose is recommended before adding adjunctive therapy. This recommendation is based on a study that showed that increasing the dose of ICS in this age group results in an improvement in asthmatic symptoms in 1- to 3-year-olds ${ }^{35}$ and a lack of data to support the use of adjunctive therapies in this age group. For other age groups, increasing the dose of ICS to medium dose or adding adjunctive therapy to a low dose of ICS is considered an equal option $^{8}$ (Figure 3). Because of a lack of comparative data, several adjunctive therapies may be considered as add-on therapy for

\section{FIGURE 3 Stepwise Approach of Managing Asthma in Youths $\geq 12$ Years of Age and Adults}

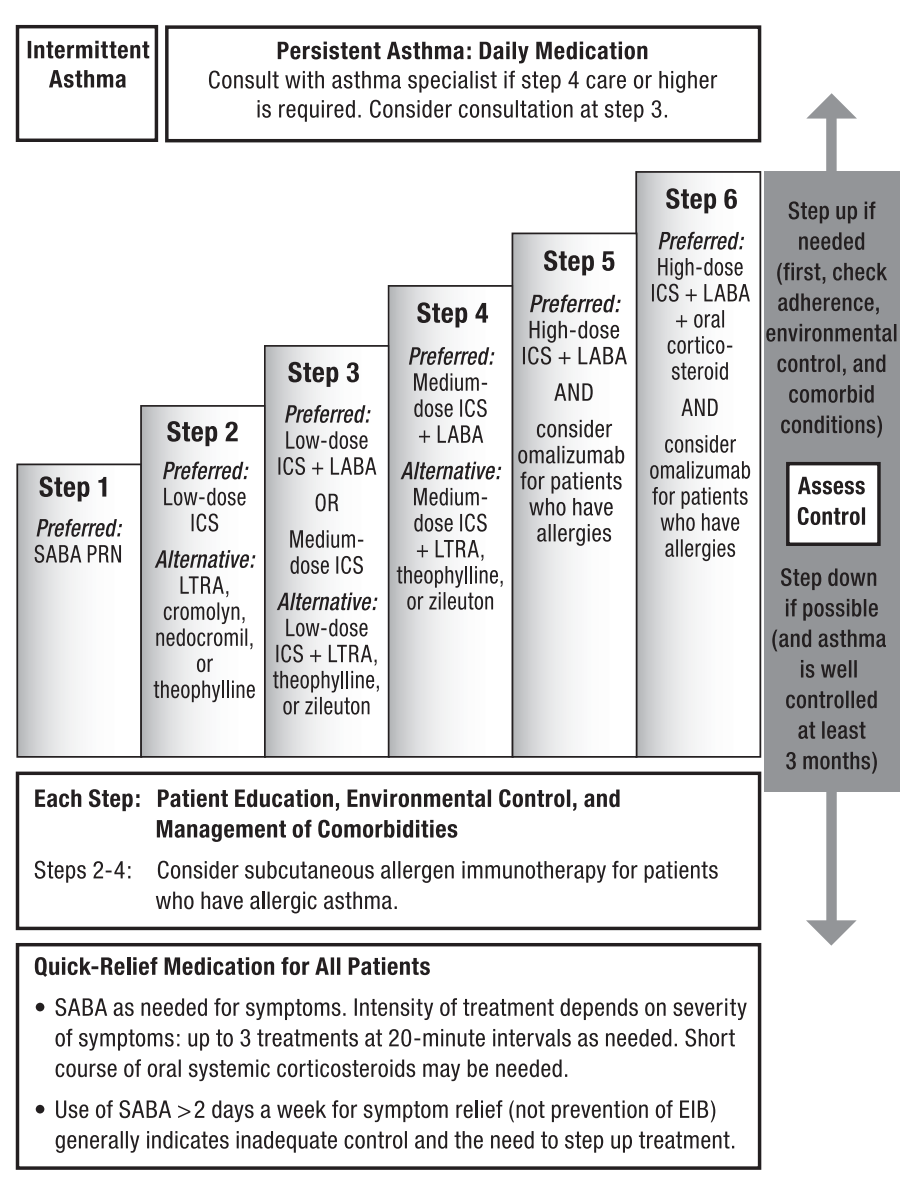

Abstracted from Figure 4-5, Expert Panel Report 3; $343 .{ }^{8}$

$E I B=$ exercise-induced brochospasm; ICS = inhaled corticosteroid;

$L A B A=$ long-acting beta-2 agonist; $L T R A=$ leukotriene receptor antagonist;

$P R N=$ as needed; $S A B A=$ short-acting beta-2 agonist.

the patient uncontrolled on low-dose ICSs, including LABAs, leukotriene receptor antagonists (LTRAs) (such as montelukast), and theophylline. While the data are not strong, of these choices, LABAs are preferred by EPR-3 on the basis of studies that show that addition of a LABA to an ICS improve lung function and symptom control. ${ }^{36-37}$

An additional change to the stepwise approach in youths and adults $\geq 12$ years is the addition of omalizumab as an option for therapy in patients who are uncontrolled on a high-dose ICS and LABA and have a demonstrated sensitivity to perennial allergens (Figure 3). Since such therapy is placed at steps 5 and 6 of the algorithm and because of the risk associated with the use of omalizumab, consultation with an asthma specialist is recommended for patients who require this step of therapy. ${ }^{8}$ 


\section{Effective Control Includes Managing Special Situations}

In addition to inherent variability in the course of asthma, adjustments to therapy may be required based on additional factors, including special situations. EPR-3, as well as other versions of the guidelines, discusses exercise-induced bronchospasm (EIB), surgery and asthma, pregnancy, and racial and ethnic disparities in asthma as 4 special situations that must be considered in the comprehensive management of the patient with asthma.

EIB is characterized by cough, dyspnea, chest pain or tightness, wheezing, or endurance problems during exercise and in some patients may be the only manifestation of asthma. All patients with asthma should be queried to determine if they experience EIB, since EIB may represent inadequately controlled asthma. Comprehensive management of EIB includes use of long-term controller therapy (if appropriate) and pretreatment before exercise with any of a number of asthma therapies, including short-acting beta-2 agonists (SABAs), LABAs, ${ }^{38}$ or LTRAs. ${ }^{39}$

Patients with asthma who undergo surgery may be at increased risk for respiratory complications. ${ }^{6,8}$ Accordingly, EPR-3 recommends that patients with asthma have a preoperative evaluation that includes review of symptoms, present medication use, and objective measurement of lung function. Attempts should be made to improve the lung function before surgery, if possible. Finally, stress-dose corticosteroids may be considered for patients who have received oral systemic corticosteroids during the past 6 months and for selected patients on a long-term high dose of an ICS. Studies have shown that if a patient's asthma is well controlled, the risk of perioperative complications is low. ${ }^{40}$

Maintenance of adequate asthma control in pregnant patients is well known to be important for both the health of the mother and the child. ${ }^{41}$ To achieve this control, EPR-3 recommends that several actions be carried out, including routine monitoring of asthma status during all prenatal visits; use of albuterol as the preferred SABA when required; use of ICS, and specifically budesonide, as the preferred long-term controller medication when one is required; and use of intranasal corticosteroids to treat concomitant allergic rhinitis, if present. Data suggest that the outcome of most mothers with asthma and their newborn infants is usually favorable, particularly if the women's asthma is well controlled during pregnancy. ${ }^{42}$

As with many other conditions, racial and ethnic disparities may influence asthma management. Studies have shown that minorities are less likely to use anti-inflammatory and preventive medications for asthma ${ }^{43}$ and are also less likely to pursue adequate follow-up care for asthma. ${ }^{44}$ This is likely due, in part, to socioeconomic barriers. Additionally, minorities are more likely to live in urban areas where a high exposure to indoor allergens (such as cockroaches) is present. Efforts to eliminate racial disparities in asthma care are underway. ${ }^{45}$

\section{Managing Exacerbations Is an Important Part of Asthma Care}

Asthma exacerbations are defined as episodes of progressively worsening dyspnea, cough, wheezing, and chest tightness (or any combination thereof) and are characterized by decreases in expiratory airflow that can be documented and quantified by spirometry. The burden of such exacerbations is substantial, with approximately 1.5 million ED visits for asthma in 1995, of which 20\%-30\% required hospital admission. ${ }^{46}$ Accordingly, prevention of asthma exacerbations is very important, and this topic has been addressed in previous versions of the asthma guideline and again in EPR-3.

Early treatment of asthma exacerbations is the most effective approach to management. Early treatment includes patient education, recognition of early signs and symptoms of an exacerbation, appropriate intensification of therapy, removal or withdrawal of any offending environmental substance, and ongoing communication between patient and clinician. ${ }^{8}$ EPR-3 updates the existing asthma guideline by simplifying the classification of asthma exacerbation into mild, moderate, severe, and life-threatening and by applying peak flow cutoff points for each of the classifications.

Management of asthma exacerbations includes therapies that can be delivered in the home and those used in urgent or emergency care. Home management includes increasing inhaled SABA use and, in some cases, adding a short course of oral systemic corticosteroids. EPR-3 removes the recommendation that suggests that an appropriate therapeutic option for home management of an asthma exacerbation is doubling the dose of ICS, on the basis of data that show this practice is ineffective. $^{47}$

Urgent or emergent management of an asthma exacerbation includes use of oxygen, SABAs, systemic corticosteroids, and consideration of adjunctive treatments in certain clinical circumstances. During this time, ongoing monitoring is vital, and once the patient is discharged, adequate follow-up is important. Studies have shown inconsistent results on the effectiveness of facilitated follow-up from the ED on asthma outcomes, but interventions such as appointment assistance have been shown to significantly increase the likelihood that discharged asthma patients will obtain primary care follow-up. ${ }^{48}$

EPR-3 makes several recommended changes to the existing asthma guideline regarding management of asthma exacerbations. First, levalbuterol is added as a potential treatment for asthma exacerbations. Second, for prehospital management (i.e., in the ambulance), standing orders for SABAs and protocols are suggested to improve airflow before the patient reaches the ED. Such protocols have been shown to be safe and effective. ${ }^{49}$ Third, magnesium sulfate and heliox are added as potential adjunctive therapy for asthma exacerbations for patients in the ED unresponsive to initial therapy. 


\section{Conclusion}

The recent release of the EPR-3 update from the NAEPP has provided managed health care professionals with new information to improve the care of patients with asthma. More comprehensive definitions of severity, including the domains of current impairment and future risk, as well as an increased focus on achieving asthma control will result in better asthma management protocols within managed care organizations (MCOs) by allowing for more precise asthma classification in accordance with improved knowledge of asthma pathophysiology and assessment. EPR-3 provides a wealth of scientific literature to refer to when constructing MCO algorithms and guidelines for asthma management. EPR-3 is lengthy, and further revision to NAEPP guidelines for asthma diagnosis and management will be necessary as knowledge about this disease increases and more pharmacologic therapies become available. Until the next update, EPR-3 represents the best of what is available to improve the care of patients with asthma.

\section{Author}

FRANK L. URBANO, MD, FACP, is medical director, Professional Resources in Management Education, Inc. (PRIME), Tamarac, Florida.

AUTHOR CORRESPONDENCE: Frank L. Urbano, MD, Medical Director, PRIME, 8201 West McNab Rd., Tamarac, FL 33321. Tel.: 954.718.6055; Fax: 954.718.6013; E-mail:f.urbano@primeinc.org

\section{DISCLOSURES}

The meeting from which this article was derived was funded by an educational grant from Genentech/Novartis Pharmaceuticals. All panel participants received compensation for travel expenses and honoraria for the 2-day meeting. The author discloses no bias or conflict of interest relating to this article.

Members of the discussion panel of asthma experts convened on June 7-8, 2007, in Hallandale, Florida.

\section{Michael Baxley, MD, MS, MPH \\ Chief Medical Officer \\ Senior Marketing Medical Executive \\ Cigna South Florida \\ Sunrise, Florida}

\section{Kathryn Blake, PharmD}

Clinical Research Scientist

Center for Clinical Pediatric Pharmacology

Nemours Children's Clinic

Jacksonville, Florida
Craig A. Jones, MD

Director of Vermont Blueprint for Health

Montpelier, Vermont

\section{H. William Kelly, PharmD, BCPS, FCCP}

Professor Emeritus of Pediatrics and Pharmacy

University of New Mexico Health Sciences Center

Albuquerque, New Mexico

\section{Daren Knoell, PharmD, FCCP}

Associate Professor of Pharmacy, Medicine and Medical Pharmacology

Ohio State University

David Heart and Lung Research Institute

Columbus, Ohio

\section{Todd A. Lee, PharmD, PhD}

Senior Investigator

Midwest Center for Health Services and Policy Research

Hines Veterans Affairs Medical Center

Hines, Illinois

Research Assistant Professor

Institute of Healthcare Studies and Division of General Internal

Medicine

Department of Medicine

Northwestern University Feinberg School of Medicine

Chicago, Illinois

\section{Bruce Sherman, MD}

Board-Certified Pulmonologist

Medical Director, Global Services

The Goodyear Tire and Rubber Company

Director, Health and Productivity Initiatives

Employers Health Coalition of Ohio

Shaker Heights, Ohio

\section{E. Rand Sutherland, MD, MPH}

Associate Professor of Medicine

Director, Carl and Hazel Felt Laboratory for Adult Asthma Research

Medical Director, Pulmonary Physiology Services

National Jewish Medical \& Research Center

University of Colorado at Denver Health Sciences Center

Denver, Colorado

\section{Michael Wechsler, MD}

Associate Director

Brigham and Women's Asthma Research Center

Assistant Professor

Harvard Medical School

Boston, Massachusetts 


\section{REFERENCES}

1. National Center for Health Statistics. Fast stats A to Z. Available at: www.cdc.gov/nchs/fastats/asthma.htm. Accessed September 19, 2007.

2. Centers for Disease Control and Prevention. National vital statistics reports. Deaths: preliminary data for 2004. Available at: www.cdc.gov/nchs/ data/nvsr/nvsr54/nvsr54_19.pdf. Accessed September 19, 2007.

3. American Lung Association. Trends in asthma morbidity and mortality. July 2006. Available at: www.lungusa.org/atf/cf/\%7B7A8D42C2-FCCA4604-8ADE-7F5D5E762256\%7D/ASTHMA06FINAL.PDF. Accessed September 19, 2007.

4. Asthma in America: a landmark survey. Executive summary. Available at: www.asthmainamerica.com/missing.html. Accessed September 19, 2007.

5. Boulet LP, Phillips R, O’Byrne P, Becker A. Evaluation of asthma control by physicians and patients: comparison with current guidelines. Can Respir J. 2002;9(6):417-23

6. National Heart, Lung, and Blood Institute. Expert panel report 2: guidelines for the diagnosis and management of asthma (EPR-2 1997). Available at: www.nhlbi.nih.gov/guidelines/archives/epr-2/asthgdln_archive. pdf. Accessed September 19, 2007.

7. National Heart, Lung, and Blood Institute. Expert panel report: guidelines for the diagnosis and management of asthma-update on selected topics 2002. Available at: www.nhlbi.nih.gov/guidelines/archives/epr-2_upd/ asthmafullrpt_archive.pdf. Accessed September 19, 2007.

8. National Heart, Lung, and Blood Institute. Expert panel report 3: guidelines for the diagnosis and management of asthma-full report 2007. August 28, 2007. Available at: www.nhlbi.nih.gov/guidelines/asthma/ asthgdln.pdf. Accessed August 29, 2007.

9. Li JTC, O'Connell EJ. Clinical evaluation of asthma. Ann Allergy Asthma Immunol. 1996;76(1):1-13.

10. Clark NM, Gong M, Schork MA, et al. Long-term effects of asthma education for physicians on patient satisfaction and use of health services. Eur Respir J. 2000;16(1):15-21.

11. Burrows B, Martinez FD, Halonen M, Barbee RA, Cline MG. Association of asthma with serum IgE levels and skin-test reactivity to allergens. N Engl J Med. 1989;320:271-77.

12. Sears MR, Burrows B, Flannery EM, Herbison GP, Hewitt CJ, Holdaway MD. Relation between airway responsiveness and serum IgE in children in asthma and in apparently normal children. N Engl J Med. 1991;325:1067-71.

13. Jackola D, Peirson-Mullany L, Corazalla E, et al. Pathophysiological states associated with asthma and atopy. J Allergy Clin Immunol. 2001;108:S177.

14. Lester LA, Rich SS, Blumenthal MD, et al. Ethnic differences in asthma and associated phenotypes: collaborative study on the genetics of asthma. J Allergy Clin Immunol. 2001;108:357-62.

15. Bousquet J, Cabrera P, Berkman N, et al. The effect of treatment with omalizumab, an anti-IgE antibody, on asthma exacerbations and emergency medical visits in patients with severe persistent asthma. Allergy. 2005; 60:302-08

16. Soler M, Matz J, Townley R, et al. The anti-IgE antibody omalizumab reduces exacerbations and steroid requirement in allergic asthmatics. Eur Respir J. 2001;18(2):254-61.

17. Busse W, Corren J, Lanier BQ, et al. Omalizumab, anti-IgE recombinant humanized monoclonal antibody, for the treatment of severe allergic asthma. J Allergy Clin Immunol. 2001;108(2):184-90.

18. Humbert M, Beasley R, Ayres J, et al. Benefits of omalizumab as add-on therapy in patients with severe persistent asthma who are inadequately controlled despite best available therapy (GINA 2002 step 4 treatment): INNOVATE. Allergy. 2005;60(3):309-16.

19. U.S. Food and Drug Administration. FDA alert: Omalizumab information. 2/2007, updated 7/2007. Available at: www.fda.gov/cder/ drug/infopage/omalizumab/default.htm. Accessed September 20, 2007.
20. National Heart, Lung and Blood Institute. Evidence table 11 Pharmacologic therapy: inhaled corticosteroids_combination therapy. Available at: www.nhlbi.nih.gov/guidelines/asthma/evid_tbls/11_ icscombther.pdf. Accessed September 20, 2007

21. Castle W, Fuller R, Hall J, Palmer J. Serevent nationwide surveillance study: comparison of salmeterol with salbutamol in asthmatic patients who require regular bronchodilator treatment. BMJ. 1993;306(6884):1034-37.

22. Nelson HS, Weiss ST, Bleecker ER, Yancey SW, Dorinsky PM. The Salmeterol Multicenter Asthma Research Trial: a comparison of usual pharmacotherapy for asthma or usual pharmacotherapy plus salmeterol. Chest. 2006;129(1):15-26.

23. U.S. Food and Drug Administration. Public health advisory: Serevent Diskus (salmeterol xinafoate inhalation powder), Advair Diskus (fluticasone propionate \& salmeterol inhalation powder), and Foradil Aerolizer (formoterol fumarate inhalation powder). Updated 5/2006. Available at: www.fda.gov/cder/drug/advisory/LABA.htm. Accessed September 20, 2007.

24. Antonicelli L, Bucca C, Neri M, et al. Asthma severity and medical resource utilisation. Eur Respir J. 2004;23(5):723-29.

25. Diette GB, Krishnan JA, Wolfenden LL, Skinner EA, Steinwachs DM, Wu AW. Relationship of physician estimate of underlying asthma severity to asthma outcomes. Ann Allergy Asthma Immunol. 2004:93(6):546-52.

26. Connolly CK, Mamun M, Alcock SM, Prescott RJ. The Darlington and Northallerton Prospective Asthma Study: best function predicts mortality during the first 10 years. Respir Med. 1998;92(11):1274-80.

27. Belessis Y, Dixon S, Thomsen A, et al. Risk factors for an intensive care unit admission in children with asthma. Pediatr Pulmonol. 2004;37(3):201-09.

28. Eisner MD, Katz PP, Lactao G, Iribarren C. Impact of depressive symptoms on adult asthma outcomes. Ann Allergy Asthma Immunol. 2005;94(5):566-74.

29. Adams RJ, Smith BJ, Ruffin RE. Factors associated with hospital admissions and repeat emergency department visits for adults with asthma. Thorax. 2000;55(7):566-73

30. Bacharier LB, Strunk RC, Mauger D, White D, Lemanske RF Jr, Sorkness CA. Classifying asthma severity in children: mismatch between symptoms, medication use, and lung function. Am J Respir Crit Care Med. 2004;170(4):426-32.

31. Fuhlbrigge AL, Weiss ST, Kuntz KM, Paltiel AD. Forced expiratory volume in 1 second percentage improves the classification of severity among children with asthma. Pediatrics. 2006;118(2):e347-55

32. Bateman ED, Frith LF, Braunstein GL. Achieving guideline-based asthma control: does the patient benefit? Eur Respir J. 2002;20:588-95.

33. Vollmer WM, Markson LE, O'Connor E, Frazier EA, Berger M, Buist AS. Association of asthma control with health care utilization: a prospective evaluation. Am J Respir Crit Care Med. 2002;165(2):195-99.

34. Bateman ED, Boushey HA, Bousquet J, et al. for the GOAL Investigators Group. Can guideline-defined asthma control be achieved? The Gaining Optimal Asthma Control study. Am J Resp Crit Care Med. 2004;170:836-44.

35. Bisgaard H, Gillies J, Groenewald M, Maden C. The effect of inhaled fluticasone propionate in the treatment of young asthmatic children: a dose comparison study. Am J Respir Crit Care Med. 1999;160(1):126-31.

36. Russell G, Williams DA, Weller P, Price JF. Salmeterol xinafoate in children on high dose inhaled steroids. Ann Allergy Asthma Immunol. 1995; 75(5):423-28.

37. Zimmerman B, D'Urzo A, Berube D. Efficacy and safety of formoterol Turbuhaler when added to inhaled corticosteroid treatment in children with asthma. Pediatr Pulmonol. 2004;37(2):122-27.

38. Richter K, Janicki S, Jorres RA, Magnussen H. Acute protection against exercise-induced bronchoconstriction by formoterol, salmeterol and terbutaline. Eur Respir J. 2002;19(5):865-71. 
39. Moraes TJ, Selvadurai H. Management of exercise-induced bronchospasm in children: the role of leukotriene antagonists. Treat Respir Med. 2004; 3(1):9-15.

40. Warner DO, Warner MA, Barnes RD, et al. Perioperative respiratory complications in patients with asthma. Anesthesiology. 1996;85(3):460-67.

41. National Heart, Lung, and Blood Institute. Working group report on managing asthma during pregnancy: recommendations for pharmacologic treatment. Available at: www.nhlbi.nih.gov/health/prof/lung/asthma/astpreg/ astpreg_full.pdf. Accessed September 20, 2007.

42. Rey E, Boulet LP. Asthma in pregnancy. BMJ. 2007;334:582-85.

43. Lieu TA, Lozano P, Finkelstein JA, et al. Racial/ethnic variation in asthma status and management practices among children in managed Medicaid. Pediatrics. 2002;109(5):857-65.

44. Shields AE, Comstock C, Weiss KB. Variations in asthma care by race/ ethnicity among children enrolled in a state Medicaid program. Pediatrics. 2004;113(3 pt 1):496-504.
45. National Institutes of Health. Strategic research plan and budget to reduce and ultimately eliminate health disparities. Vol. I: Fiscal years 2002-2006. Bethesda, MD: National Institutes of Health; 2004. Available at: http://ncmhd.nih.gov/our_programs/strategic/pubs/VolumeI_031003EDrev. pdf. Accessed September 21, 2007.

46. Rodrigo G, Rodrigo C, Hall JB. Acute asthma in adults. Chest. 2004; 125:1081-102

47. Fitzgerald JM, Becker A, Sears MR, Mink S, Chung K, Lee J. Doubling the dose of budesonide versus maintenance treatment in asthma exacerbations. Thorax. 2004;59(7):550-56.

48. Baren JM, Boudreaux ED, Brenner BE, et al. Randomized controlled trial of emergency department interventions to improve primary care follow-up for patients with acute asthma. Chest. 2006;129(2):257-65.

49. Markenson D, Foltin G, Tunik M, Cooper A, Treiber M, Caravaglia K. Albuterol sulfate administration by EMT-basics: results of a demonstration project. Prehosp Emerg Care. 2004;8(1):34-40 